\title{
Integration of homeostatic signaling and food reward processing in the human brain
}

\author{
Joe J. Simon, ${ }^{1,2}$ Anne Wetzel, ${ }^{1}$ Maria Hamze Sinno, ${ }^{1}$ Mandy Skunde, ${ }^{1}$ Martin Bendszus, ${ }^{3}$ \\ Hubert PreissI, ${ }^{4}$ Paul Enck, ${ }^{5}$ Wolfgang Herzog, ${ }^{1}$ and Hans-Christoph Friederich ${ }^{1,2}$ \\ 'Department of General Internal Medicine and Psychosomatics, Centre for Psychosocial Medicine, University Hospital \\ Heidelberg, Heidelberg, Germany. ${ }^{2}$ Department of Psychosomatic Medicine and Psychotherapy, Medical Faculty, Heinrich- \\ Heine-University Düsseldorf, Düsseldorf, Germany. ${ }^{3}$ Department of Neuroradiology, University Hospital Heidelberg, \\ Heidelberg, Germany. ${ }^{4}$ Institute for Diabetes Research and Metabolic Diseases, Helmholtz Center Munich, University of \\ Tübingen, Tübingen, Germany; German Center for Diabetes Research (DZD e.V.), Neuherberg, Germany; Internal Medicine VI \\ and Institute of Pharmaceutical Sciences, Department of Pharmacy and Biochemistry, Eberhard Karls Universität Tübingen, \\ Tübingen, Germany; Institute for Diabetes and Obesity, Helmholtz Diabetes Center, Helmholtz Center Munich, University \\ of Tübingen, Tübingen, Germany; and German Research Center for Environmental Health GmbH, Neuherberg, Germany. \\ ${ }^{5}$ Department of Psychosomatic Medicine and Psychotherapy, University Medical Hospital, Tübingen, Cermany.
}

BACKCROUND. Food intake is guided by homeostatic needs and by the reward value of food, yet the exact relation between the two remains unclear. The aim of this study was to investigate the influence of different metabolic states and hormonal satiety signaling on responses in neural reward networks.

METHODS. Twenty-three healthy participants underwent functional magnetic resonance imaging while performing a task distinguishing between the anticipation and the receipt of either food- or monetary-related reward. Every participant was scanned twice in a counterbalanced fashion, both during a fasted state (after $\mathbf{2 4}$ hours fasting) and satiety. A functional connectivity analysis was performed to investigate the influence of satiety signaling on activation in neural reward networks. Blood samples were collected to assess hormonal satiety signaling.

RESULTS. Fasting was associated with sensitization of the striatal reward system to the anticipation of food reward irrespective of reward magnitude. Furthermore, during satiety, individual ghrelin levels were associated with increased neural processing during the expectation of food-related reward.

CONCLUSIONS. Our findings show that physiological hunger stimulates food consumption by specifically increasing neural processing during the expectation (i.e., incentive salience) but not the receipt of food-related reward. In addition, these findings suggest that ghrelin signaling influences hedonic-driven food intake by increasing neural reactivity during the expectation of food-related reward. These results provide insights into the neurobiological underpinnings of motivational processing and hedonic evaluation of food reward.

TRIAL REGISTRATION. ClinicalTrials.gov NCT03081585.

FUNDING. This work was supported by the German Competence Network on Obesity, which is funded by the German Federal Ministry of Education and Research (FKZ 01GI1122E).

Conflict of interest: The authors have declared that no conflict of interest exists.

Submitted: January 24, 2017

Accepted: June 23, 2017

Published: August 3, 2017

\section{Reference information:}

JCI Insight. 2017;2(15):e92970.

https://doi.org/10.1172/jci.

insight. 92970 
Specifically, mesocorticolimbic reward pathways have been identified as important neural components influencing our eating patterns. The neural processing of food rewards can be divided into the desire to eat food prompted via incentive motivation ("wanting"), i.e., the assignment of incentive values to food-related cues, and the perceived "pleasure" during the receipt of food reward ("liking," ref. 1). Although still disputed, these components are thought to be subserved by separable neural substrates that can be partially manipulated and measured in an independent fashion (2). Incentive delay tasks are commonly used to investigate these mechanisms in human subjects, since this paradigm allows separation of both phases.

Activity in the striatum, a key region of the mesolimbic reward network, has been consistently observed during the anticipation of both monetary- and food-related rewards (3, 4). Furthermore, this region is involved in the control of food intake and interacts closely with the signal of energy homeostasis (5). Food-specific reactivity in this region has been related to success in weight-loss programs (6), and, in a previous study from our group, we observed a relation between food-related anticipatory ventral striatal activation and impaired hormonal satiety signaling (7). On the other hand, activation in the orbitofrontal cortex (OFC) is often found when receiving rewards and has been ascribed a role in reward evaluation (8). The lateral OFC (1OFC) has been described as the secondary gustatory cortex (9); activation in this region has been linked to the current reward values of tastes $(10,11)$, subjective feeling of appetite $(12)$, and individual BMI levels (13-15). Repetitive transcranial magnetic stimulation of the left $1 \mathrm{OFC}$ has been found to reduce the desire for food consumption (16). Finally, the medial OFC (mOFC) is relevant for the evaluation of food reward (17) and has been related to the subjective experiences of pleasantness (18). In a previous report, we found an association between activation in the mOFC during the receipt phase of the food incentive delay (FID) task and bulimic-type eating disorders (19).

Physiological hunger is considered to increase the sensitivity of these circuits to food-related stimuli (20) via homeostatic gut-brain signaling (21). However, the exact interaction between homeostatic states and the two phases of neural food reward still remains unknown. It has been observed that the reinforcing value of electrical stimulation of the brain reward network is enhanced during a state of physiological hunger (22), which suggests a general stimulatory effect on reward perception (21). Furthermore, both dopaminergic as well as opioid signaling, which are crucial during the processing of the anticipation or receipt of rewards, respectively (23), have been found to be affected by homeostatic satiety signaling (5, 24-26). However, studies investigating neural reactivity to food stimuli found both baseline as well as reward-level-dependent homeostasis-induced increases in dopamine (27-29) and opioid signaling (30, 31). Therefore, it still remains unclear whether mesolimbic reward signaling during a fasted state is generally increased irrespective of reward modality or if it is only increased for food reward. Furthermore, even if physiological hunger only increases reactivity to food stimuli, it remains unknown if this increase is contingent with reward level, i.e., fasting induces stronger reactions to cues indicating higher reward but not to cues indicating no reward, or if reactivity of the brain reward network to food reward is elevated during fasting in a "hyperactive general fashion," i.e., it reacts strongly to all levels of food reward.

Ghrelin is a brain-gut peptide that plays an important role in energy homeostasis and the regulation of hunger (32). Furthermore, it is well-known to regulate rewarding aspects of eating, thereby increasing food-seeking behavior $(33,34)$. It has been found that intravenous administration of ghrelin increases the incentive value of food cues (35) and ghrelin has been associated with alcohol cravings (36), indicating a role of ghrelin in more "general" reward processing. However, the exact role of ghrelin in feeding behavior as well as hedonic processing of food cues still remains unclear (32). Previous studies found that postprandial reductions in ghrelin are associated with reduced signaling in reward-related areas of the brain (37). Furthermore, during satiety, ghrelin levels have been found to be related to hedonic eating, both in normal weight (38) as well as obese individuals (39), which points to a role of ghrelin in the "consumption of food for pleasure" when sated (40).

To investigate the influence of fasting on specific subcomponents of food reward processing, we used functional magnetic resonance imaging (fMRI) to compare blood oxygen level-dependent (BOLD) activity during the processing of food- and monetary-related rewards between different physiological states in healthy women. Since previous studies mostly delivered food reward in a passive manner (41) or used different task designs for different types of reward, we employed both a monetary-related as well as a food-related incentive delay task and used the same task design for both conditions to be able to directly compare both types of reward (Figure 1$)$. We previously showed $(7,15,19)$ that the fMRI task employed in this study is able to probe typical, both food and monetary, reward-related brain regions. The monetary reward condi- 
tion was used to assess whether satiety status would influence reward processing independently of reward modality, i.e., induce a "general" increase of brain reward functioning. Participants were scanned twice in a counterbalanced fashion, once after having fasted for 24 hours and once immediately after lunch. To increase the specificity of our analyses, we focused on predefined regions of interest (ROIs) in the ventral striatum (VS) as well as 1OFC and mOFC. Since ghrelin has been discussed as a potential "link" between fasting and increased reward behavior (42) by stimulating the mesolimbic reward circuitry (34), and has been found to enhance the response to food stimuli in various brain areas involved in the processing of food reward (43), we decided to primarily focus on ghrelin signaling.

We focused on the VS during the expectation phase, since this region has been consistently related to the anticipated value of a rewarding outcome $(44,45)$. During the reward receipt, we focused on regions within the OFC, notably the $\mathrm{mOFC}$, which has been previously related to subjective pleasantness ratings during food consumption (8), and the 1OFC, which has been described as part of the secondary gustatory cortex (9). To examine the influence of individual differences in hormonal hunger signaling on neural processing, we investigated the association between brain activation and individual ghrelin levels. Finally, to investigate differences between satiety states in reward modality-independent interactions in the mesolimbic reward network, we performed a resting-state functional connectivity analysis. Previous studies investigating this issue observed conflicting results, with some studies observing connectivity differences in the brain reward network between satiety states (46) while others did not (47). These observed differences have been discussed as a potential biological marker for obesity (48).

\section{Results}

Subjects. Twenty-three healthy controls were enrolled in the study. Demographic and clinical characteristics are provided in Table 1. Hormonal satiety parameters derived from the participants' blood samples prior to measurement as well as hunger ratings confirmed their satiety status (Table 2).

Behavioral performance. Reaction times during the FID task were influenced by reward level $(F(1,22)$ $=8.89, P=0.001, n=23$, repeated-measures ANOVA) but not by metabolic state $(F(1,22)=2.69, P$ $=0.115)$. When expecting 10 snack points (SP), participants reacted quicker when hungry than when sated $(t(23)=-2.268, P=0.033,2$-tailed $t$ test $)$. Reaction times during the monetary incentive delay (MID) task were also influenced by reward level $(F(1,21)=13.10, P<0.001, n=22)$ but not by metabolic state $(F(1,21)=0.47, P=0.499)$.

Imaging data - expectation of food reward. We found that phasic, i.e., stimulus-dependent activity in the VS was related to reward level during the expectation of both food- and monetary-related rewards (Figure 2A and Figure 3A). Furthermore, we observed a tonic increase (i.e., independent of reward level) in the VS when expecting food reward during the fasted state compared with satiety. Figure 4 illustrates the interaction effect between satiety status and reward level during the expectation phase. For food reward, lines for both the left and right VS are parallel, which indicates no interaction between satiety state and reward level. The main effect of satiety state (i.e., tonic increase) is revealed in the vertical distance (magnitude of percentage signal change) between the two lines, and the main effect of reward level is revealed in the slope of each line.

Expectation of monetary reward. The anticipation of monetary-related reward in the VS was not influenced by satiety state. As displayed in Figure 4, the slope of each line also revealed a main effect of reward level, but there were no significant vertical differences between the two lines (i.e., no significant effect of satiety level).

Receipt of food reward. Phasic activity in the mOFC and 1OFC was influenced by reward level during the receipt of food reward, but we did not observe a tonic increase in activity during the fasted state (Figure 2B and Figure 5).

Receipt of monetary reward. The receipt of monetary reward was not related to satiety state and only related to reward level for the mOFC (Figure 3B). However, we observed an interaction between satiety state and activation in the $\mathrm{mOFC}$ during the receipt of monetary reward. Figure 5 indicates that while there was no interaction for the 1-EUR and 20-cent conditions, we observed that fasting apparently increases brain activation for the 0-EUR condition. However, a follow-up $t$ test revealed no significant difference between fasted and satiated state (paired $t$ test: $P=0.308$ ). The interaction between reward type (food- vs. monetary-related reward) and metabolic state (fasted state vs. sated state) remained insignificant for all OFC ROIs (all $P$ values $>0.463$ ). 
Table 1. Participant characteristics

\begin{tabular}{|c|c|}
\hline & $\begin{array}{c}\text { Mean } \pm \text { SD } \\
\text { (minimum to maximum) }\end{array}$ \\
\hline$n$ total & 23 \\
\hline Age (yr) & $27.9 \pm 5.6(20.6-46.7)$ \\
\hline BMI $\left(\mathrm{kg} / \mathrm{m}^{2}\right)$ & $21.1 \pm 1.9(18.04-25)$ \\
\hline Education (yr) & $16.2 \pm 1.4(12-17)$ \\
\hline Maximal lifetime weight (kg) & $64.4 \pm 6.6(55-78)$ \\
\hline MWT-B & $30.5 \pm 2.5(26-36)^{A}$ \\
\hline EDE-Q & $0.6 \pm 0.5(0-1.9)$ \\
\hline BDI-II & $3.5 \pm 3.2(0-10)^{A}$ \\
\hline \multicolumn{2}{|c|}{$\begin{array}{l}\text { MWT-B, Vocabulary-Based Test for the Assessment of } \\
\text { Premorbid Intelligence; EDE-Q, Eating Disorder Examination } \\
\text { Questionnaire; BDI-II, Beck Depression Inventory. }{ }^{\text {AD Data missing }} \\
\text { for one person. }\end{array}$} \\
\hline
\end{tabular}

Differences between food and monetary reward processing. To statistically assess differences between brain activation during the processing of food and monetary reward, we performed a 3-way ANOVA with reward modality, satiety state, and reward level as independent variables and percentage signal change as the dependent variable. There was no significant 3-way interaction either during the expectation of reward (right and left VS) or during the receipt of reward in the right OFC and $1 O F C$. Only the mOFC displayed a significant 3-way interaction (mOFC, $F(2,42)=3.39, P=0.043$, $n=22$ ) during the receipt of reward. However, the interaction between modality and reward level as well as between modality and satiety state in the mOFC was not significant $(F(2,42)$ $=2.43, P=0.1, F(2,42)=0.06, P=0.939$,

respectively). To further examine potential differences between modalities, we also looked at the interaction between modality and satiety state (i.e., independently of reward level). We found a significant interaction for the right and left VS $(F(1,21)=4.86, P=0.039$ and $F(1,21)=5.05, P=0.035$, respectively) but not for the OFC ROIs (all $P$ values $>0.463$ ). However, post-hoc tests (2-sample $t$ tests) revealed no significant differences between modalities for the right and left VS (all $P$ values $>0.056$ ).

Correlational analyses. When investigating the relation between brain activation and hormonal satiety parameters, we found a positive correlation between VS activation during the expectation of a high food reward (10 SP) and ghrelin levels in the satiated state (Figure 6A). Furthermore, we found that maximal lifetime weight was negatively related to VS activation during the expectation of a high food reward (10 SP) in the fasted state (Figure 6B).

Functional connectivity analysis. To determine the influence of satiety state on the spontaneous organization of the reward network, we performed a resting-state functional connectivity analysis. We performed a spatial group independent component analysis (ICA) and employed a spatial correlation to identify the reward network from the resulting components (see Figures 7 and 8). However, we failed to observe an increased connectivity within the brain reward network during the fasted state when compared with satiety (Figure 8).

\section{Discussion}

To our knowledge, this is the first study directly comparing reward-related brain activation between different satiety states during the processing of food and monetary rewards using an incentive delay task. Our main finding indicates that fasting induced a reward-level independent increase of neural processing during the expectation but not the receipt of food-related reward. This hyperactivation of the reward system was specific to food, as we observed no metabolic influence on monetary reward processing and resting-state connectivity of the brain reward network. Finally, individual ghrelin levels were related to increased food reward processing in the VS when sated.

During the expectation of rewards, satiety status had a differential effect on food reward processing when compared with monetary reward. This observation does not support the assumption of a general modality-independent increase of neural reward signaling during a fasted state (21). Although previous studies observed an influence of satiety status on monetary reward processing (49) as well as dysfunctional neuronal processing of monetary reward in participants with obesity (50), our results point to a food reward-specific sensitization of neural reward networks during an energy-deficient state. Since we only employed monetary reward as a second reward modality, whether other types of rewards also remain unaffected by satiety status still has to be assessed.

However, when fasted, brain activation was increased for all food reward levels. This indicates that physiological hunger increases reactivity to food-related stimuli irrespective of their hedonic value but is in contrast to some previous studies, which found that physiological hunger increases reward processing only for high-calorie 
Table 2. Hormonal satiety parameters and hunger rating

\begin{tabular}{|c|c|c|c|c|c|}
\hline & $\begin{array}{c}\text { Fasted } \\
(\text { mean } \pm \text { SD) }\end{array}$ & $\begin{array}{c}\text { Satiated } \\
\text { (mean } \pm \text { SD) }\end{array}$ & $t$ & $d f$ & $P$ \\
\hline Ghrelin total (fmol/ml) $(n=13)$ & $936.1 \pm 522.3$ & $617.5 \pm 277.6$ & 3.7 & 12 & 0.003 \\
\hline $\operatorname{PYY}(\mathrm{pg} / \mathrm{ml})(n=14)$ & $50.1 \pm 13.4$ & $126.8 \pm 37.2$ & -7.5 & 13 & $<0.001$ \\
\hline Glucose (mg/dl) $(n=23)$ & $79.1 \pm 8.9$ & $95.7 \pm 23.7$ & -3.3 & 22 & 0.003 \\
\hline Leptin $(\mu \mathrm{g} / \mathrm{ml})(n=23)$ & $5.6 \pm 4.1$ & $9 \pm 5.6$ & -5.7 & 22 & $<0.001$ \\
\hline Insulin $(\mathrm{mU} / \mathrm{ml})(n=23)$ & $5.3 \pm 3.2$ & $38.4 \pm 27.8$ & -5.7 & 22 & $<0.001$ \\
\hline Free fatty acids (mmol/l) $(n=23)$ & $1.1 \pm 0.3$ & $0.3 \pm 0.3$ & 8.8 & 22 & $<0.001$ \\
\hline $\begin{array}{l}\text { Hunger rating before measurement } \\
(n=22)\end{array}$ & $6.6 \pm 2.1$ & $1.1 \pm 1.3$ & 12.1 & 21 & $<0.001$ \\
\hline $\begin{array}{l}\text { Hunger rating after measurement } \\
(n=22)\end{array}$ & $7.7 \pm 1.9$ & $1.6 \pm 1.8$ & 11.9 & 21 & $<0.001$ \\
\hline $\begin{array}{l}\text { Mood rating before measurement } \\
(n=22)\end{array}$ & $6.8 \pm 1.9$ & $7.2 \pm 0.9$ & -1.7 & 21 & 0.1 \\
\hline $\begin{array}{l}\text { Mood rating after measurement } \\
(n=22)\end{array}$ & $6.5 \pm 1.9$ & $6.6 \pm 1.6$ & 0.05 & 21 & 0.962 \\
\hline
\end{tabular}

stimuli, whereas satiety increases reward processing of low-calorie stimuli (20). This may be in part due to the fact that previous studies either used a passive delivery of food reward (for an overview, please see ref. 41) or performed a comparison between different stimuli types or different levels of reward during hunger (43), which in effect normalizes the observed signal, so that a general reward level-independent increase in reward processing cannot be assessed. Furthermore, most previous studies were unable to directly compare reward modalities due to differences in task design (for example, Dean and colleagues, ref. 51, or Stice and colleagues, ref. 52). It is unclear if physiological hunger increases reactivity to food-related stimuli irrespective of their hedonic value.

The results furthermore suggest a modulatory effect of physiological hunger during expectation of food reward but not during reward outcome evaluation. Specifically, it influenced "wanting" for food by promoting a general increase of activation irrespective of reward level, whereas it had no effect on the "liking" of food. Our results highlight the importance of mesolimbic dopaminergic signaling during a state of physiological hunger by stimulating food consumption via increased incentive motivation. Specifically, together with previous observations of increased neural reactivity in the striatum when anticipating food during physiological hunger $(53,54)$, it appears that food consumption in an energy-deficient state is mainly driven by an increased motivational processing of food-related cues. Our findings question the old saying that "hunger is the best spice" (55) and suggest that, in line with the saying "the sizzle sells the steak," hunger is a motivator for food consumption irrespective of food type and palatability. The fact that hedonic reward evaluation during the receipt was not affected by satiety status is an interesting observation, considering overeating in the scope of obesity in which individuals eat beyond their homeostatic needs. Opioid signaling, which is strongly involved in hedonic processing during the receipt of rewards (21), is thought to enhance pleasure experienced during eating irrespective of food type (56) via an enhancement of palatability and suppression of satiety signals (57). This indicates that overeating could be facilitated by a rewarding effect of food that remains unaffected by the satiety status.

We also found that the higher the lifetime BMI, the lower the motivational food reward processing in the VS. Dysfunctional brain reward activation is a common observation in obese subjects (58), highlighting the importance of altered homeostatic and hedonic interactions in obesity. However, obesity is commonly related to increased activation during anticipatory food reward $(6,59)$, and it has been postulated that, similar to drug addiction, obesity is in part caused by a sensitization of mesolimbic reward networks, leading to an abnormally increased incentive motivation for food-related reward (60).

Our observation that individual differences in ghrelin when satiated specifically influence the "wanting" of food $(27,61)$ is in line with previous observations in which injection of ghrelin in the VTA in rats increases preference for and motivation to obtain highly palatable food (62). Furthermore, in ghrelin receptor-deficient mice, cue potentiated feeding is disrupted and meal anticipatory behavior is reduced $(63,64)$, highlighting the importance of ghrelin signaling for feeding driven by external food-related cues (65), which has also been observed 

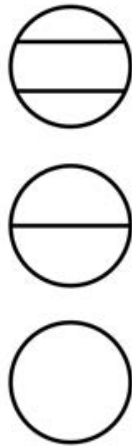

Cue

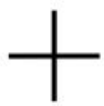

Delay

$3 \mathrm{~s}$

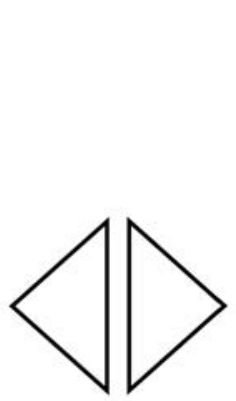

FID
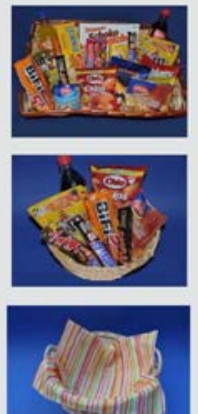

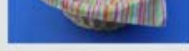

Feedback $1.5 \mathrm{~s}$

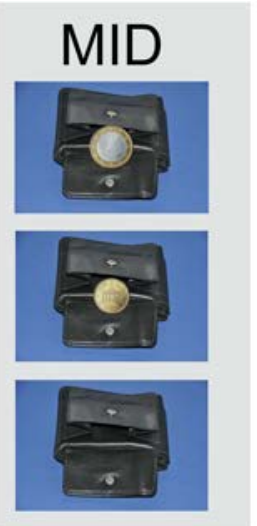

Target

$1 \mathrm{~s}$

Figure 1. Incentive delay task. At the beginning of each trial, participants were presented with a cue depicting the amount of money (monetary incentive delay task) or snack points (SP; food incentive delay task) to be won. A circle with two horizontal lines represented 1 EUR or 10 SP, a circle with one line represented 0.2 EUR or 2 SP, and an empty circle represented 0 EUR or 0 SP. After a delay period, participants had to react by pressing a left or right button according to the position of the triangle. During the feedback phase, participants were informed about the amount won during the respective trial. Immediately after the fMRI scan, participants received the amount of money won and were able to choose snacks from a basket according to the amount of SP won.

in individuals with obesity (39). Since ghrelin influenced brain reward processing in the sated state, our results add to the notion of ghrelin as an important contributor to hedonic eating where food is consumed due to its rewarding properties and not to maintain energy homeostasis (38).

A number of limitations have to be noted. We aimed to increase the specificity of our analyses by focusing on central hubs of the brain network relevant for the processing food reward (15). However, it is important to acknowledge the fact that our choice of ROIs was not comprehensive, as additional brain regions are involved in food reward processing. Furthermore, since we compared resting-state connectivity maps during different satiety levels using SPM8, we relied on random-field theory corrections to control the family-wise error rate, which can produce a very high degree of false positives, especially when employing a low cluster forming threshold (66). Although this has not affected our results since we observed no significant results, even at our rather lenient threshold, this has to be noted as a limitation of this study. Additionally, it has previously been shown that neural reward processing in women is influenced by the menstrual cycle (67), which was not controlled for in this study. This is a potential confounding aspect and should be addressed in future studies. Furthermore, since we employed abstract food stimuli, our observations may differ from tasks that include the receipt of actual taste stimuli during an fMRI scan. Although we previously found that our task was able to probe brain regions typically associated with the processing of food-related reward (3), and we aimed at investigating differences between reward modalities without the potential confounding effect of differing experimental protocols, this is a further limitation of this study. Finally, although the concepts of "wanting" and "liking" have become an integral part of research investigating neural food processing (68), the brain regions involved in these processes are still to some extent uncertain. For example, Berridge and colleagues found that subregions of the nucleus accumbens subserve bot motivational and hedonic processing of rewards $(69,70)$. However, investigating subcomponents of the nucleus accumbens with fMRI is not feasible due to restrictions in spatial resolution. When taking all of the evidence into account, one may conclude that, although striatal activation may account for both "wanting" and "liking," when observing it during the anticipation phase of the task, it may be valid to state a causal relation with motivational aspects of reward processing (see for example, refs. 71, 72).

Taken together, we show for the first time to our knowledge in humans that "wanting" and "liking" of food are differentially related to satiety states, providing a framework for future neurobiological research in the field of obesity and eating disorders. Our results add to the common observation that human eating behavior is closely related to reward processing in mesocorticolimbic reward circuits (73). 
A

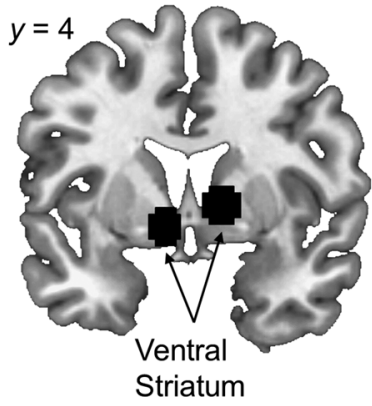

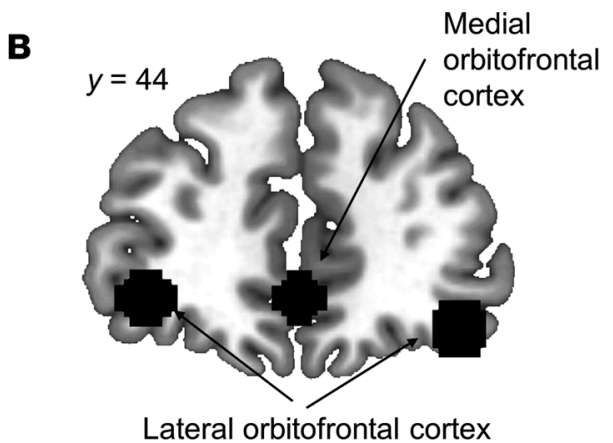

Lateral orbitofrontal cortex
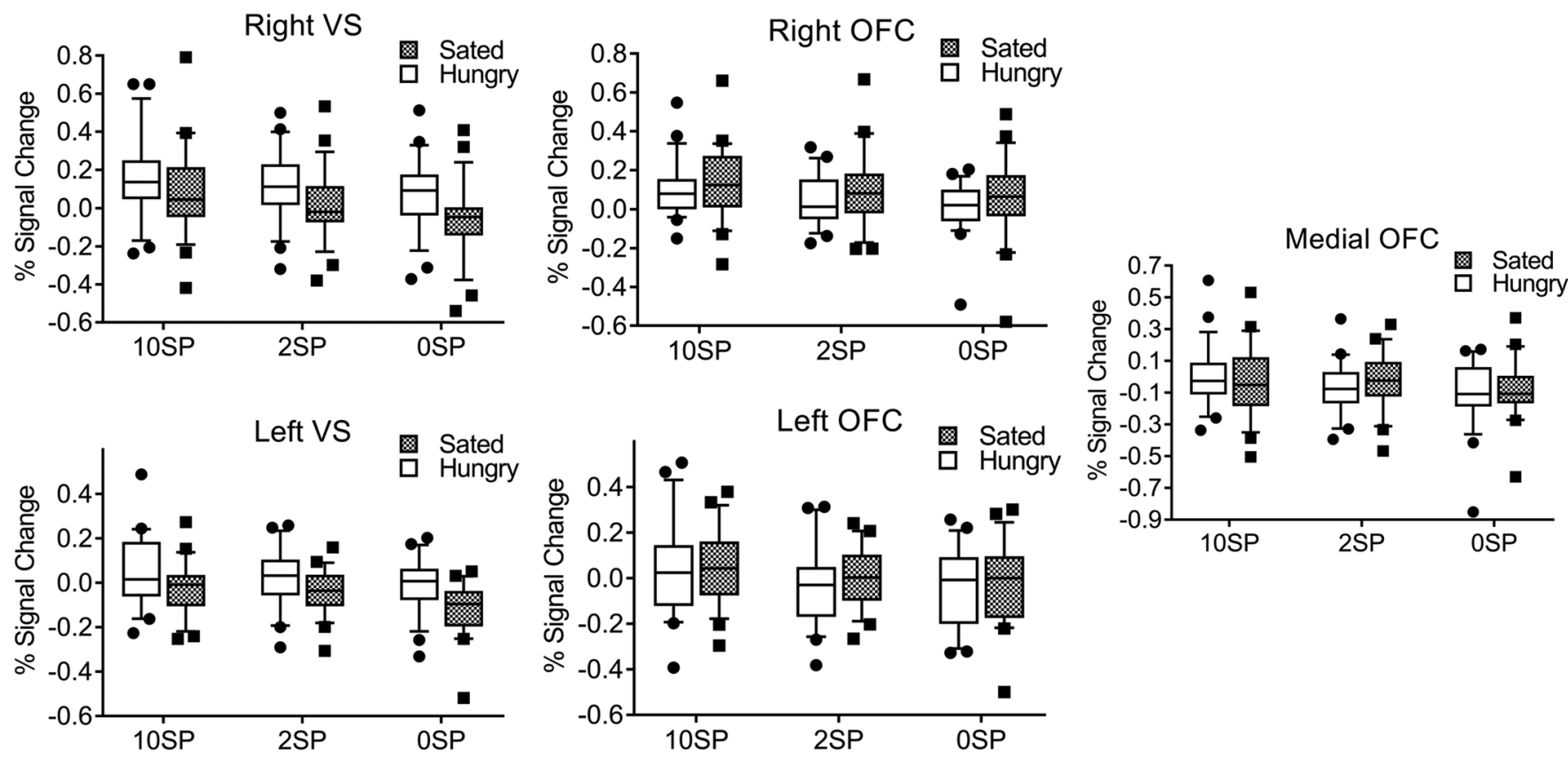

Figure 2. Neural activation during the expectation and receipt of food reward is differentially related to satiety status. (A) During the expectation of food-related reward, percentage signal change extracted from the right and left ventral striatum was influenced by reward level $(F(1,22)=13.17, P<0.001$, $F(1,22)=12.1, P<0.001$, respectively, $n=23)$ as well as satiety state $(F(1,22)=5.09, P=0.034, F(1,22)=6.78, P=0.016$, respectively, $n=23)$. We observed no interaction between reward level and satiety state (all $P$ values $>0.35$ ). (B) During the receipt of food-related reward, percentage signal change extracted from the right, left, and medial orbitofrontal cortex was influenced by reward level $(F(1,22)=6.36, P=0.004, F(1,22)=8.66, P=0.001, F(1,22)=4.83, P=$ 0.013 , respectively, $n=23$ ) but not by satiety state (all $P$ values $>0.2, n=23$ ). We observed no interaction between reward level and satiety state (all $P$ values $>0.17$ ). Repeated-measures ANOVAs were used for the statistical analysis. In box-and-whisker plots, horizontal bars indicate the medians, boxes indicate 25th to 75 th percentiles, and whiskers indicate 10th and 90th percentiles.

\section{Methods}

\section{Participants}

Twenty-five healthy women took part in the study (Table 1). All participants underwent the Structured Clinical Interview for the DSM-IV (SCID) (74) and filled out the Beck Depression Inventory (75). All participants were right handed, female, over the age of 18 years, and were required to have a BMI of between $18 \mathrm{~kg} / \mathrm{m}^{2}$ and $25 \mathrm{~kg} / \mathrm{m}^{2}$. Exclusion criteria included claustrophobia, metallic implants, pregnancy, and lifetime diagnoses of eating disorder, bipolar disorder, borderline personality disorder, psychosis, and alcohol or drug abuse. Furthermore, participants currently dieting or experiencing significant weight fluctuations over the past 9 months were excluded. Participants were recruited via advertisements and flyers. Two participants had to be excluded due to an unusual high error rate during the experimental tasks ( $Z s$ > 3). Participants received a fixed reimbursement for their participation in the study (100 EUR) in addition to the amount of money (maximal 60 EUR) and SP (maximal 600 SP) won during both time points. 
A

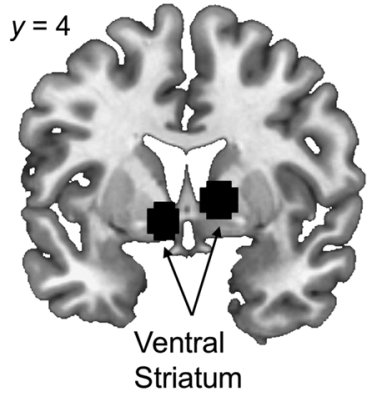

B

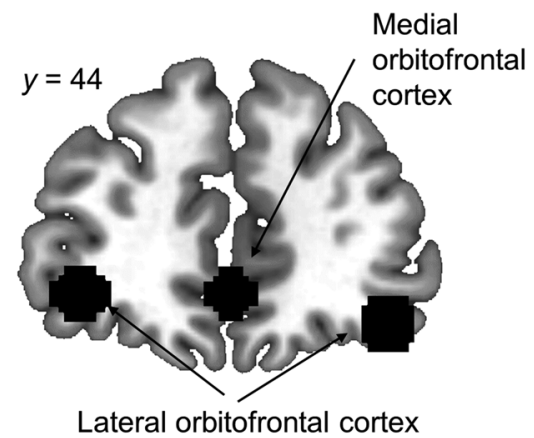

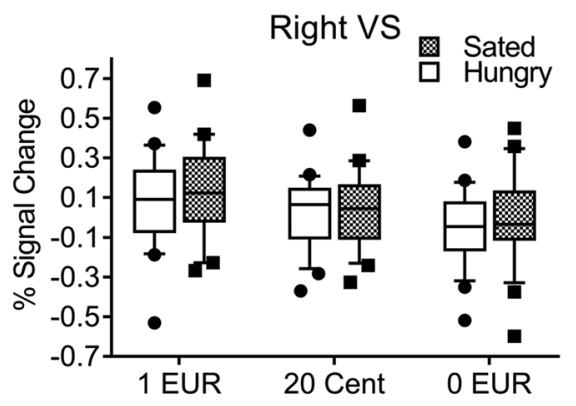
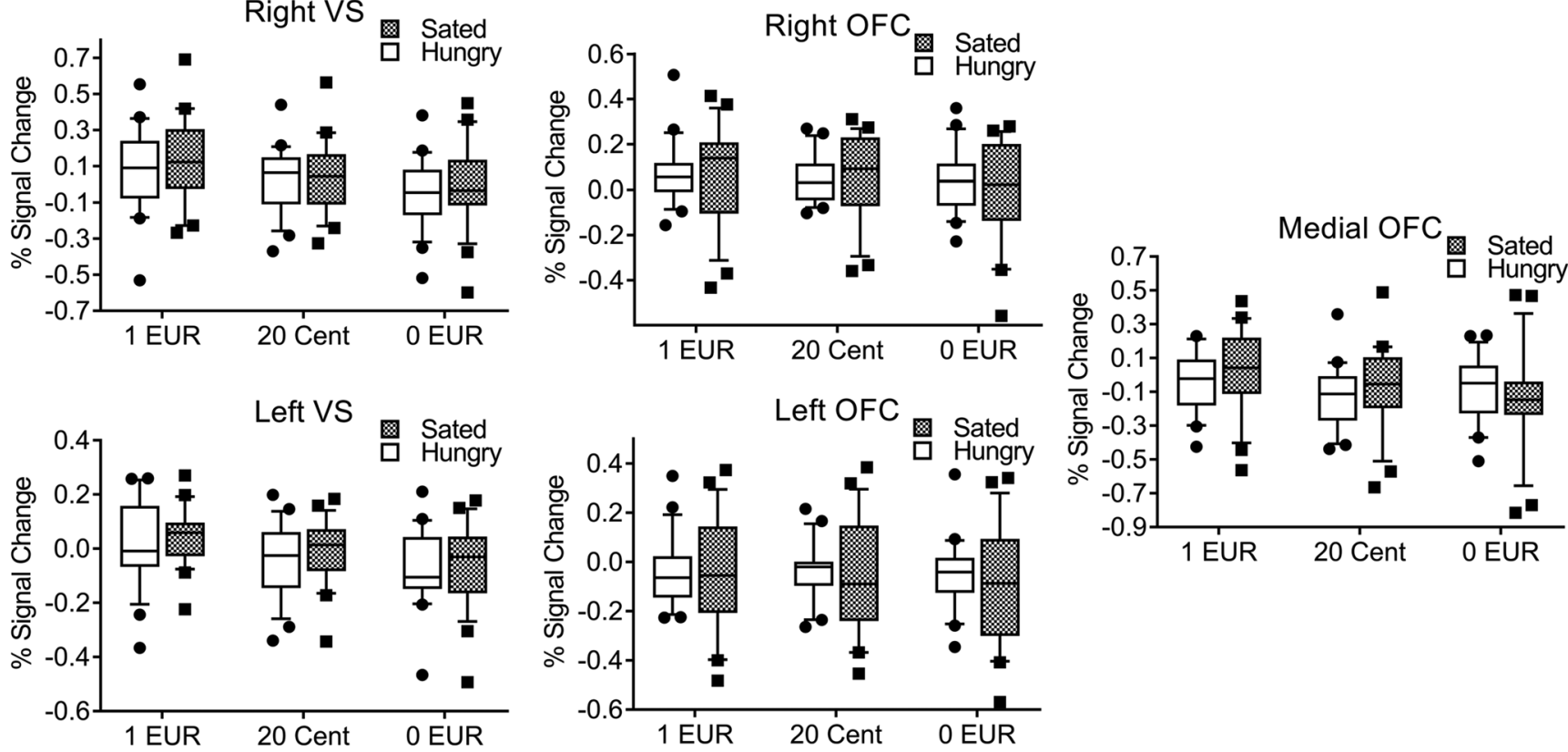

Figure 3. Neural activation during monetary reward processing. (A) During the expectation of monetary-related reward, percentage signal change extracted from the right and left ventral striatum was influenced by reward level $(F(1,21)=21.47, P<0.001, F(1,21)=28.06, P<0.001, n=22$, respectively) but not by satiety state (all $P$ values $>0.47, n=23$ ). We observed no interaction between reward level and satiety state (all $P$ values $>0.47, n=23$ ).

(B) During the receipt of food-related reward, percentage signal change extracted from the right and left orbitofrontal cortex was neither influenced by reward level nor by satiety state (all $P$ values $>0.09, n=23$ ). Activity in the medial orbitofrontal cortex was influence by reward level $(F(1,21)=12.36, P<$ $0.001, n=23)$ but not by satiety state $(P=0.81, n=23)$. We observed an interaction effect between reward level and satiety state in the medial orbitofrontal cortex $(F(2,42)=4.26, P=0.021, n=23$ ) but the right or left orbitofrontal cortex (all $P$ values $>0.34, n=23$ ). Repeated-measures ANOVAs were used for the statistical analysis. In box-and-whisker plots, horizontal bars indicate the medians, boxes indicate 25 th to 75 th percentiles, and whiskers indicate 10th and 90th percentiles.

\section{Procedure}

Participants were scanned twice on two different days in a counterbalanced fashion, once after having fasted for 24 hours and once immediately after lunch. The mean interval between measurements was 10.2 days $(\mathrm{SD}=5.8$ days). Every scan occurred at lunch time, starting at 12:00 a.m. and ending at 2:00 p.m. During the fasted state, participants were asked to refrain from eating anything for 24 hours prior to the measurement, though they could drink water or herbal tea. They were instructed to eat lunch as usual around 11:00 and 12:00 a.m. on the previous day. During the satiety condition, participants received a standardized meal containing approximately $650 \mathrm{kcal} 1$ hour prior to scanning. Participants also received a meal after measurement during the fasting condition. The order of the two experimental sessions was randomized: half of the participants first underwent the fasting session and the other half participated first in the satiety session (within-subject cross over design). To verify the satiety status of participants, we assessed serum glucose, insulin, free fatty acids levels, and peptide tyrosine tyrosine (PYY), a hormone essential in the reduction (76) of hunger (Table 2). There was a significant difference among all four parameters when comparing fasting and satiety values (all $P$ values $<0.003$, Table 2), indicating that all participants complied with the fasting 


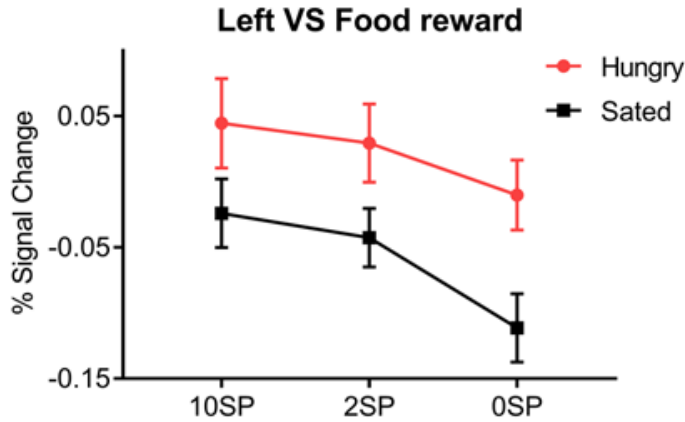

\section{Left VS monetary reward}

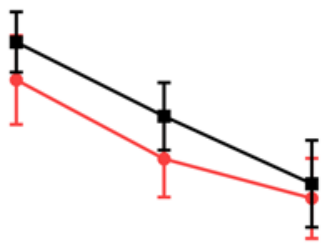

1 EUR 20 Cent 0 EUR

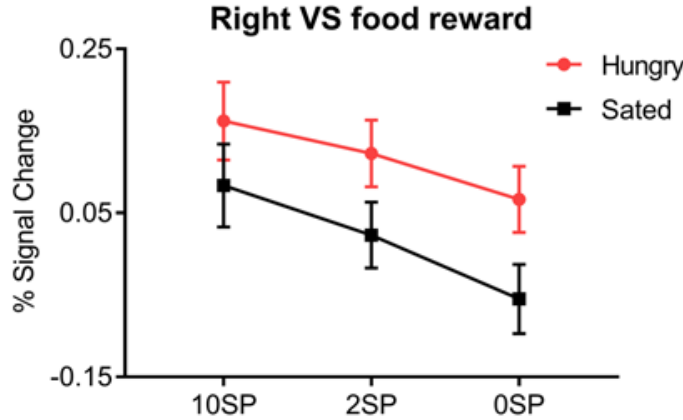

\section{Right VS monetary reward}

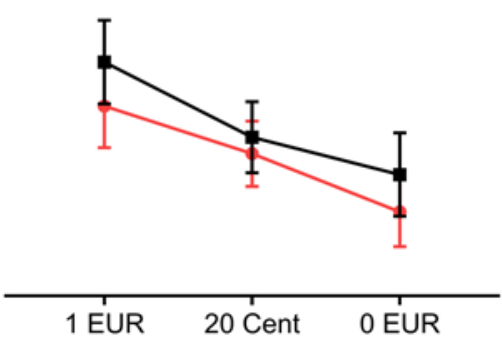

Figure 4. Interaction analyses during the expectation of reward. The top row shows the percentage signal change extracted from the left VS during the expectation of food-related reward and monetary-related reward during the fasted state (red line with circles) and the sated state (black line with squares) divided according to reward level (as indicated on the $x$ axis). The bottom row shows the signal change extracted from the right VS during the expectation of food- and monetary-related reward during different satiety states. Error bars indicate SEM.

instruction. Prior to scanning, participants were presented with a basket containing all the foods they could trade for the SP to be won during the FID task (Table 3).

All psychometric evaluations and the SCID interview occurred during the sated condition. Participants filled out a number of self-report and demographic questionnaires and were asked about their eating and dieting behaviors. Specifically, we employed a Vocabulary-Based Test for the Assessment of Premorbid Intelligence (77), the Eating Disorder Examination Questionnaire (78), the Maudsley Obsessive-Compulsive Inventory (79), and the Barrat Impulsivity Scale (80).

\section{Biochemical analysis of hormonal parameters}

On both days, blood samples were taken shortly before the fMRI measurement (at around 11:45 a.m.) After the blood was centrifuged at $4^{\circ} \mathrm{C}$, the serum was separated and stored at $-80^{\circ} \mathrm{C}$. Insulin and leptin were measured using materials mentioned later in the sentence based on a sandwich ELISA assay from Merck Millipore (Merck KGA; ghrelin, catalog EZGRT-89K; insulin, catalog EZHIASF-14K; leptin, catalog EZHL-80SK; PYY, catalog EZHPYYT66K), with a detection limit of $0.85 \mu \mathrm{U} / \mathrm{ml}$ and $0.2 \mathrm{ng} / \mathrm{ml}$, respectively. For ghrelin and PYY analyses, Pefabloc (Sigma-Aldrich) and DPP4 inhibitors (Sigma-Aldrich) were added immediately to the blood. Additionally, for ghrelin analyses, serum was acidified to protect the active form of ghrelin. Therefore, the ghrelin total values contain both acyl and des-acyl ghrelin. Each sample was measured in duplicate. The intra-assay coefficients of variation for insulin were $2.58 \%$ and $2.19 \%$ at concentrations of 7.75 and $45.63 \mu \mathrm{U} / \mathrm{ml}$, respectively. The inter-assay coefficients of variations at these concentrations were below $4 \%$ and $12 \%$, respectively. For leptin, the intra-assay coefficients of variations were $0.17 \%$ and $3.16 \%$ at concentrations of 2.52 and $15.82 \mathrm{ng} / \mathrm{ml}$, respectively. The inter-assay coefficients of variations at these concentrations were below $21 \%$ and $10 \%$, respectively. The intra-assay coefficients of variation for total ghrelin were $3.4 \%$ and $4.9 \%$ at concentrations of 509.54 and $2,053.5 \mu \mathrm{U} / \mathrm{ml}$, respectively. The inter-assay coefficients of variations at these concentrations were below $3 \%$ and $4 \%$, respectively. The intra-assay coefficients of variation for PYY were $8.6 \%$ and $5.4 \%$ at concentrations of 135.65 and 499.31 $\mu \mathrm{U} / \mathrm{ml}$, respectively. The inter-assay coefficients of variations at these concentrations were below $11 \%$ and 


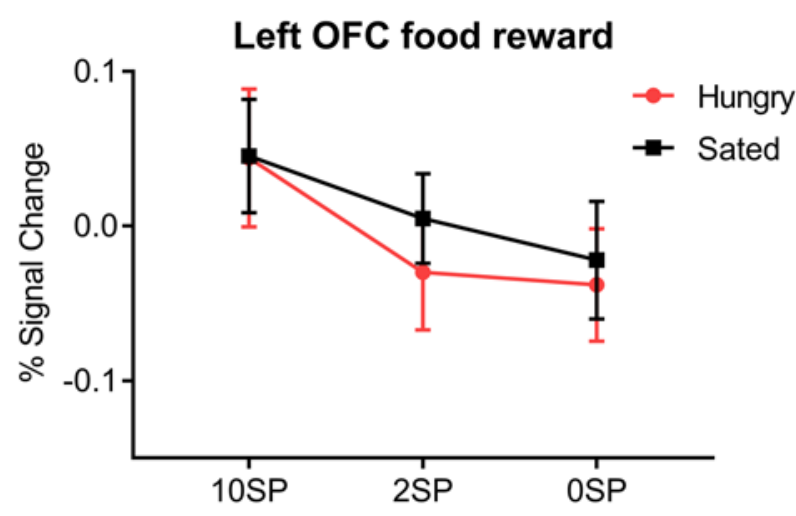

\section{Left OFC monetary reward}

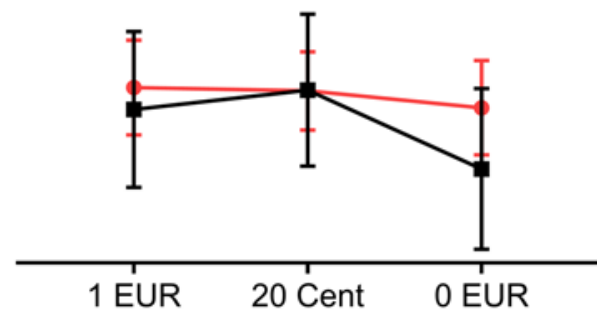

\section{Right OFC food reward}

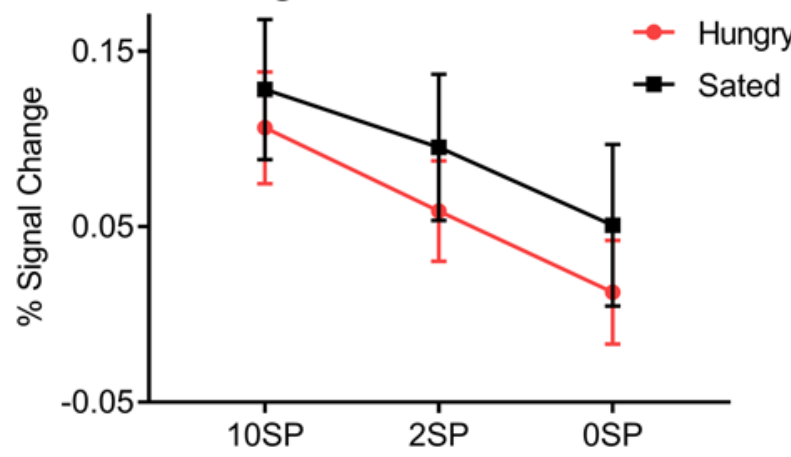

Medial OFC food reward

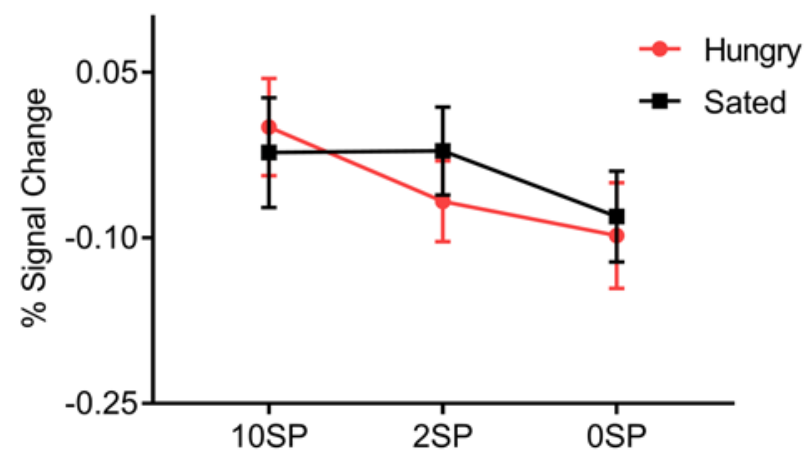

\section{Medial OFC monetary reward}

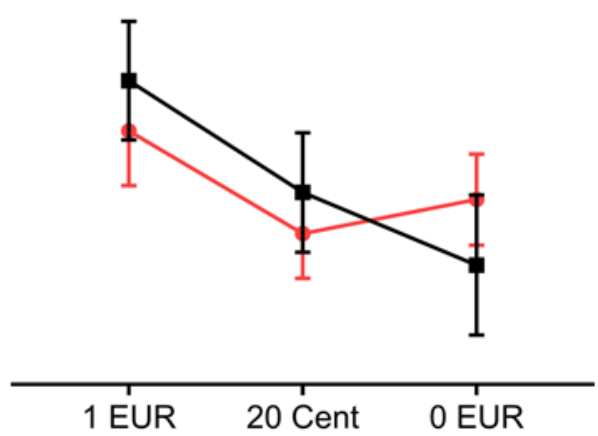

Figure 5. Interaction analyses during the receipt of reward. The top row shows the percentage signal change extracted from the left OFC during the receipt of food-related reward and monetary-related reward during the fasted state (red line with circles) and the sated state (black line with squares) divided according to reward level (as indicated on the $x$ axis). The middle row shows the signal change extracted from the right OFC during the receipt of food-and monetary-related reward during different satiety states. The bottom row shows the signal change extracted from the medial OFC during the receipt of food- and monetary-related reward during different satiety states. Error bars indicate SEM.

$6 \%$, respectively. Glucose concentrations were performed at the central laboratory of the University of Heidelberg on a Siemens Advia 2400 device using the hexokinase method. Due to technical reasons, the ghrelin values could not be evaluated for 10 participants and the PYY values could not be measured for 9 participants, leaving 13 and 14 participants for the ghrelin and PYY analyses, respectively. 

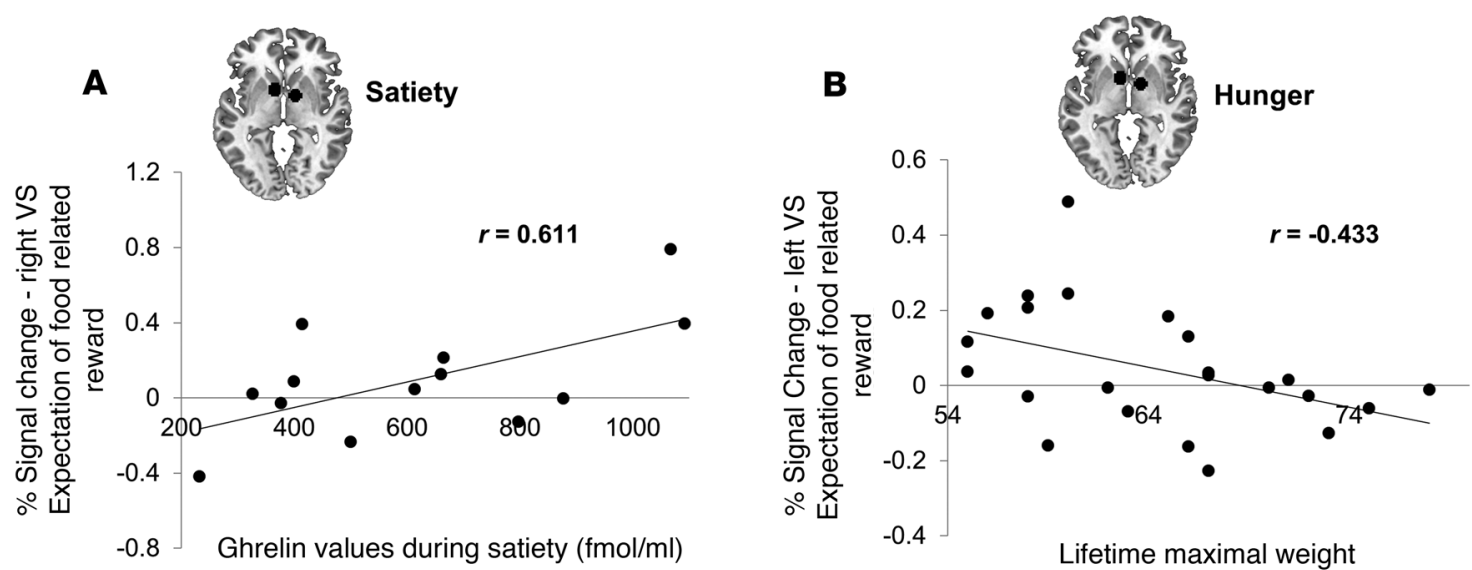

Figure 6. Neural food reward processing is related to hormonal satiety parameters and lifetime maximal weight. (A) Percentage signal change of BOLD activation extracted from the right ventral striatum during the expectation of food-related reward (high reward: 10 snack points) when satiated was positively related to ghrelin total values (Pearson $r(11)=0.611, P=0.027$, 2-tailed correlation, $n=13$ ). (B) Percentage signal change of BOLD activation extracted from the left ventral striatum during the expectation of a food-related reward (high reward: 10 snack points) during the fasted state was negatively related to lifetime maximal weight $(r(21)=-0.433, P=0.039$, 2-tailed correlation, $n=23)$.

\section{Incentive delay tasks}

We used two types of incentive delay tasks to measure monetary- and food-related reward processing, a MID task and a FID task (Figure 1). In the MID task, participants were able to win a certain amount of money, which was paid out immediately after scanning, whereas they could win SP in the FID task, which could be exchanged for sweet and salty snacks as well as beverages and fruits immediately after the MRI measurement. The use of SP allows us to directly compare food- with monetary-related rewards as well as to avoid variance caused by interindividual differences in food preferences. Both tasks differed only in the modality of reward type; task timing and structure were identical. The MID task is a well-established paradigm to probe reward-related processing (3), whereas the FID task has been found to reliably induce activity in brain regions related to the anticipation and receipt of food reward (15).

Participants completed four blocks of reward tasks consisting of 55 trials per block. The sequence was either snack-money-snack-money or money-snack-money-snack and was counterbalanced over participants. There was a short break in the middle of the sequence. Potential rewards varied over three levels that were indicated using graphical cues. Each trial lasted 6.25 seconds and started with the presentation of a symbol (duration $=750 \mathrm{~ms}$ ) indicating the potential amount of money or number of SP to be won with a correct response (i.e., 1 EUR, 0.2 EUR, or 0 EUR for the MID task and 10 SP, 2 SP, or 0 SP for the FID task). After an anticipation period (duration $=3.000 \mathrm{~ms}$ ), during which a cross hair was presented, participants had to correctly react to one of two symbols (triangle pointing to the right or to the left), with a left or right button press corresponding to the direction of the triangle (index or middle finger of the right hand) within a fixed interval of 1.000 milliseconds. The comparably long time range allocated for the reaction during the detection task resulted in a very high success rate. To guarantee a steady rate of reward versus nonreward throughout all trials, we employed a probabilistic reward pattern in which reward was not paid out in 30 predefined trials of the 80 reward trials. Following target presentation participants were presented with a feedback (duration $=1.500 \mathrm{~ms}$ ) displaying the amount of money or SP won during the respective trial and about the cumulative total. In order to increase statistical efficiency, trials were separated by jittered intertrial intervals ranging from 1 to 8 seconds, with a mean of 3.5 seconds. An incorrect button press resulted in 0 payout, whereas a penalty of -1 EUR or -10 SP was applied if participants failed to react. The maximal amount to be won during the MID task was 30 EUR and 300 SP during the FID task, with any snack of the basket being worth $50 \mathrm{SP}$, allowing a maximum of 6 snacks to be taken. Subjects were shown the money and snack basket before entering the scanner.

Before entering the scanner, participants performed a practice version of both tasks, lasting 3 minutes each, for which they received neither payment nor snacks. Additionally, participants performed a response inhibition task (unpublished observations). During scanning, participants viewed visual stimuli on a projection screen via 


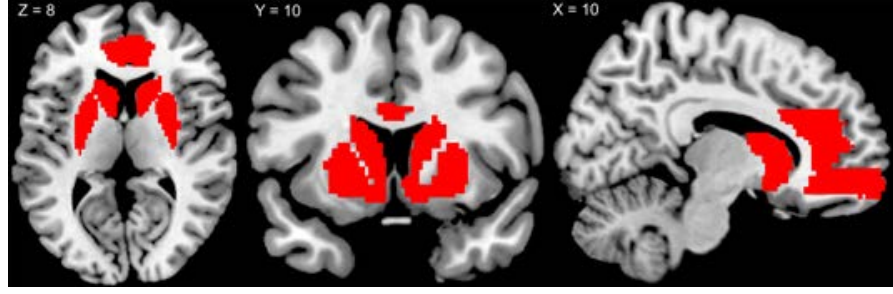

Figure 7. Anatomical brain reward mask. Anatomical mask used to identify the reward network from the group independent component analysis. The mask contained the bilateral caudatus, putamen, thalamus, anterior cingulate cortex, and medial orbitofrontal cortex. The mask was created using the Wake Forest University PickAtlas, and all regions were taken from the Automated Anatomical Labeling atlas. a mirror fixed to the head coil. The duration of each block was 9.15 minutes, with a total duration of 36.6 minutes for all four blocks.

\section{fMRI acquisition}

Images were collected using a 3-T Siemens Trio MRI scanner (Siemens Medical Solutions) equipped with a 32-channel head coil. Participant's heads were stabilized by foam padding around the head within the coil to minimize head movements. Each functional run lasted $9.15 \mathrm{~min}$ utes, with 275 volumes per run. In order to minimize susceptibility artifacts in the OFC, 30 oblique slices (interleaved acquisition) with a $10^{\circ}$ angle relative to the AC-PC axis were acquired with 1-mm interslice gap, using a T2*-sensitive single-shot EPI sequence with the following parameters: repetition time (TR) $=2,000$ milliseconds, echo time $(\mathrm{TE})=30$ milliseconds; this resulted in an in-plane resolution of $3 \times 3 \times$ $4 \mathrm{~mm}^{3}$, flip angle of $80^{\circ}$, and field of view of $192 \times 192 \mathrm{~mm}$. Furthermore, high-resolution T1 MPRAGE anatomical images were acquired (192 slices; voxel size $1 \times 1 \times 1 \mathrm{~mm}^{3} ; \mathrm{TR}=1,570 \mathrm{~ms}$; TE $=2.63 \mathrm{~ms} ; 9^{\circ}$ flip angle) for anatomical reference.

\section{Analysis of fMRI data}

Preprocessing. fMRI data were preprocessed and analyzed with SPM8 (http://www.fil.ion.ucl.ac.uk/spm/software/spm8/). To account for magnetic field equilibration, four volumes from the start of each functional run were excluded from the analysis. All functional images were inspected manually for artefacts. Functional scans were slice time corrected with reference to the first slice using SPM8's Fourier phase-shift interpolation. Images were then realigned, with the allowed motion limited to $\pm 4-\mathrm{mm}$ translation and $\pm 3^{\circ}$ of rotation over the entire experiment, and unwarped to correct for artifacts due to susceptibility-by-movement interactions. Individual $\mathrm{T} 1$ images were coregistered with the mean $\mathrm{T} 2 *$ images and subsequently segmented. Both structural and functional images were normalized to the standard anatomical Montreal Neurological Institute (MNI) space using the transformation parameters from the segmentation and bilinear interpolation as implemented in SPM, resulting in a voxel size of $3 \times 3 \times 4 \mathrm{~mm}^{3}$ for the functional images and a voxel size of $1 \mathrm{~mm}^{3}$ for the high-resolution anatomic images. Furthermore, functional images were smoothed with an 8-mm full-width half-maximum isotropic Gaussian kernel. Finally, a 128-second high-pass filter was used to remove low-frequency noise and signal drift.

Analysis. At the single-subject level, a general linear model was constructed by separately modeling regressors for the three different anticipation phases for each reward modality (i.e., anticipation of 1 EUR, 0.2 EUR, and 0 EUR and anticipation of 10 SP, 2 SP, and 0 SP) and the five different outcome phases (i.e., receipt or omission of 1 EUR, 0.2 EUR, and 0 EUR and receipt or omission of 10 SP, 2 SP and 0 SP) as explanatory variables convolved with the $\gamma$-variate function described by Cohen (81). The targets and error trials were included as regressors of no interest. Based on our a priori hypothesis, we then extracted the mean percentage signal change for each regressor using MarsBaR (82) in predefined ROIs. Our motivation to focus on ROI analysis instead of a more traditional whole-brain analysis was to increase the specificity of our analysis. We used the same task in previous studies $(7,15,19)$ and found the VS and 1 OFC to be crucial for the neural processing of food-related rewards. For the analysis of the expectation phase, we defined masks for the bilateral VS. Based on a previous fMRI study from our group (15) in which we employed the same food reward task in a sample of 27 healthy women, we created 8-mm-diameter spheres centered on the peak activation observed in this study during the anticipation of a high food-related reward compared with no food-related reward (expectation of 10 SP versus expectation of $0 \mathrm{SP}$ ) in both the right and left VS (MNI space: $x=12, y=2, z=2$, for the right VS and MNI: $x=-9, y=8, z=-6$, for the left VS). For the analysis of the receipt reward phase, we created 8-mm-diameter spheres centered on the peak activation during the receipt of a food-related reward compared with the receipt of no food-related reward (receipt of $10 \mathrm{SP}$ versus receipt of $0 \mathrm{SP}$ ) in both the right and left OFC (MNI: $x=42, y=$ $41, z=-14$, for the right OFC and MNI: $x=-39, y=47, z=-6$, for the left OFC). Due to the common observa- 


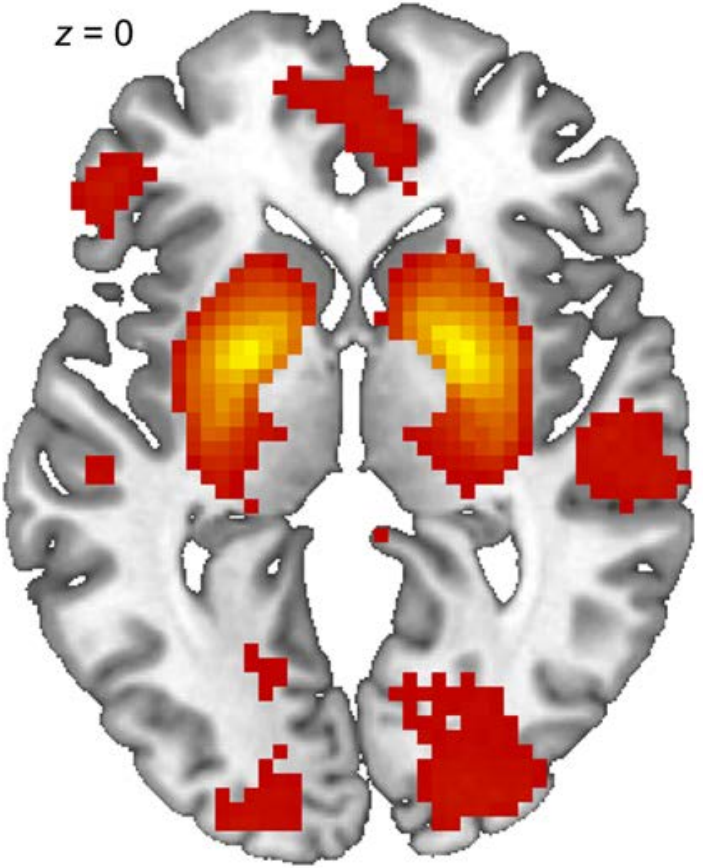

3.2
40.7
Figure 8. Reward component derived from the group independent component analysis. The brain reward network obtained using a spatial group independent component analysis and subsequent spatial correlation with an a priori defined structural mask of relevant brain reward regions (correlation with the template mask, $r=0.341)$. The obtained component was included in a random-effect analysis using a 1-sample $t$ test, with age and BMI as covariates of no interest $(n=23)$. The statistical map was thresholded at a cluster-defining threshold of $P<0.001$ uncorrected (cluster size $k>10$ ). We observed significant clusters of activation at a family-wise error corrected cluster level threshold of $P$ $<0.05$ in the bilateral striatum, posterior cingulate cortex, inferior parietal cortex, lingual gyrus, inferior and superior frontal gyrus, precuneus, medial and lateral orbitofrontal cortex, occipital cortex, and the postcentral gyrus. We observed no results when comparing component activity between metabolic states using a 2-sample $t$ test with age and BMI as covariates of no interest.

tion of mOFC activation during the evaluation of rewards (8), we constructed a 8-mm-diameter sphere centered on the peak of activation (MNI: $x=0, y=48, z=6$ ) observed in a previous study in which we analyzed neural activation during the receipt of monetary reward (83). The extracted percentage signal change was then used to perform random-effects analyses using SPSS version 22 (IBM Corp.).

At the group level, extracted percentage signal change was entered into a repeated-measures ANOVA to assess influences of metabolic state, reward modality, and reward level on brain activation. ANOVAs were performed separately for the expectation (using signal change extracted from the right and left VS) and receipt phase (using signal change extracted from the right OFC, left OFC, and mOFC). At first we performed a 3-way ANOVA with reward modality, satiety state, and reward level as independent variables, and the respective brain activation estimates (i.e., percentage signal change) as dependent variable. To specify modality-specific influences of metabolic state and reward level, we further performed 2-way ANOVAs, with metabolic state and reward level as independent and brain activation estimates as dependent variables. Correlational analyses between percentage signal change and psychometric measures as well as homeostatic parameters were performed using the Pearson product-moment correlation coefficient ( 2 tailed). Given the complex mechanisms of hunger regulation, we only investigated specific influences of ghrelin signaling when satiated.

\section{Resting-state data analysis}

Data acquisition. Before performing the event-related reward task, every participant underwent a resting-state MR acquisition lasting 5.3 minutes. 160 images were collected with the same parameters as during the task (i.e., 30 oblique interleaved slices with 1 -mm interslice gap using a $\mathrm{T} 2{ }^{*}$-sensitive single-shot EPI sequence); the only difference was that the slices were acquired parallel to the AC-PC axis (as opposed to employing a $10^{\circ}$ angle relative to the AC-PC axis as during the task). Participants were instructed to keep still with their eyes closed, not to think of anything in particular, and not to fall asleep.

\section{Data preprocessing}

Resting-state fMRI (rs-fMRI) data were preprocessed and analyzed with SPM8 (http://www.fil.ion.ucl.ac.uk/ $\mathrm{spm} /$ software/spm8/). To account for magnetic field equilibration, four volumes from the start of each functional run were excluded from the analysis. Resting-state functional images were processed using the same 
Table 3. Food items tradable for snack points

\begin{tabular}{|c|c|c|}
\hline & & $\begin{array}{l}\text { Amount included in } \\
\text { the snack basket }\end{array}$ \\
\hline \multirow[t]{6}{*}{ Salty, savory foods } & Sausage snack (BiFi, $25 \mathrm{~g}$ ) & 5 \\
\hline & Crisps (salt, $50 \mathrm{~g}$ ) & 1 \\
\hline & Salty crackers (Tuc, 100 g) & 1 \\
\hline & Salt sticks (125 g) & 1 \\
\hline & Peanuts (150 g) & 2 \\
\hline & Nut and raisin mix (125 g) & 1 \\
\hline \multirow[t]{7}{*}{ Sweet foods } & Snickers (40 g) & 5 \\
\hline & Mars (45 g) & 7 \\
\hline & Twix (58 g) & 6 \\
\hline & Cereal bar (Balisto, $18.5 \mathrm{~g}$ ) & 9 \\
\hline & Chocolate cookie (PickUp, 28 g) & 5 \\
\hline & M\&Ms (40 g) & 5 \\
\hline & Gummy bears ( $75 \mathrm{~g})$ & 4 \\
\hline \multirow[t]{3}{*}{ Fruit } & Apple & 1 \\
\hline & Banana & 1 \\
\hline & Seasonal fruit (orange or pear) & 1 \\
\hline \multirow[t]{3}{*}{ Beverages } & Water (0.5 I) & 1 \\
\hline & Coca-Cola (0.5 I) & 1 \\
\hline & Carbonated apple juice (Lift, $0.5 \mathrm{I}$ ) & 1 \\
\hline
\end{tabular}

procedure as during the event-related task: slice-time correction, realignment, and unwarping to correct for susceptibility-by-movement interactions; coregistration with mean $\mathrm{T} 2$ * images and segmentation; normalization to MNI space using the transformation parameters from the segmentation, resulting in a voxel size of $3 \times 3 \times 4 \mathrm{~mm}^{3}$; and finally images were smoothed using an 8-mm full-width half-maximum isotropic Gaussian kernel. To evaluate the quality of our rs-fMRI data, we performed a manual inspection of all images (no participant exceeded \pm 2.5 - $\mathrm{mm}$ translational movement or $\pm 2^{\circ}$ rotation over the entire 160 data points) and used the TsDiffAna toolbox (http://www.fil.ion.ucl. ac.uk/spm/ext/\#TSDiffAna) to control for data artefacts. Furthermore, we employed Artifact Detection Tools (http://www.nitrc.org/projects/artifact_detect) to identify problematic time points during the scan. An image was identified as an outlier if the head displacement exceeded a threshold of 2-mm normalized movement in any direction or if the global mean intensity threshold in the image exceeded three SDs from the mean image intensity for the entire scan. During the fasted state, the mean amount of motion outliers was $0 \pm 0$ and the mean amount of intensity outliers was $4.91 \pm 6.29$; during the satiety condition, the mean amount of motion outliers was $0 \pm 0$ and the mean amount of intensity outliers was $6.39 \pm 13.84$. Importantly, the two time-points did not differ with regard to the number of intensity-related outliers $(t(46)=-0.6, P=0.55)$.

ICA

We performed a spatial group ICA (84) using the Group ICA fMRI Toolbox (GIFT; http://mialab.mrn. org/software/) to identify temporally coherent resting-state networks. The dimensionality of the preprocessed rs-fMRI data from each subject was reduced through principal component analysis. Using the high-model-order ICA approach proposed by Allen et al. (85), the data were then decomposed into 75 spatial independent components based on the infomax algorithm (86) implemented in GIFT. We performed 20 ICA (ICASSO) to ensure stability of the estimated components. For each subject, component spatial maps were reconstructed (using GICA3, ref. 87) and converted to $z$ values.

\section{Component selection}

We employed a spatial correlation to identify the reward network from the resulting components. To this end, we employed an anatomical mask containing the bilateral caudatus, putamen, thalamus, anterior cingulate cortex, and mOFC (Figure 7). The mask was created using the Wake Forest University PickAtlas (88), and all regions were taken from the Automated Anatomical Labeling atlas (89). We chose the component with the highest correlation with the template mask $(r=0.341)$.

\section{Differences in functional connectivity between satiety states}

To assess differences between fasting and satiety in functional connectivity within the brain reward network, individual component images were included in a random-effect analysis and compared using a 2-sample $t$ test, with age and BMI as covariates of no interest. We employed a cluster-defining threshold of $P<0.001$ uncorrected (cluster size $k>10$ ) and report results significant at a family-wise error corrected cluster level threshold of $P<0.05$. Results were masked using the anatomical mask of the brain reward network.

\section{Statistics}

Analyses of event-related fMRI data were performed using SPM8 (http://www.fil.ion.ucl.ac.uk/spm/software/spm8/), brain activation estimates were extracted using MarsBaR (82) and analyzed using SPSS version 22 (IBM Corp.), resting-state MR data was analyzed using SPM8 and GIFT (http://mialab.mrn.org/ 
software/). Means of extracted percentage signal change were compared using ANOVA models: at first we performed a 3-way ANOVA with reward modality, satiety state, and reward level as independent variables and the respective brain activation estimates (i.e., percentage signal change) as dependent variable. To specify modality-specific influences of metabolic state and reward level, we further performed 2-way ANOVAs with metabolic state and reward level as independent and brain activation estimates as dependent variables. When appropriate, post-hoc tests were performed using a 2-tailed Student's $t$ test. Pearson correlations were calculated to determine the association between activation estimates and hormonal satiety parameters as well as clinical variables. $P<0.05$ was considered statistically significant.

\section{Study approval}

The present study was approved by the local ethics committee of the Medical School of the University of Heidelberg and is listed on clinicaltrials.gov (NCT03081585). All participants provided written informed consent prior to participation.

\section{Author contributions}

JJS, MB, HP, PE, WH, and HCF designed the research. AW and MS collected the data. JJS, AW, and MHS analyzed the data. JJS, MB, HP, PE, WH, and HCF wrote the manuscript.

\section{Acknowledgments}

This work was supported by the Kompetenznetz Adipositas (Competence Network on Obesity), which is funded by the German Federal Ministry of Education and Research (FKZ 01GI1122E).

Address correspondence to: Joe J. Simon, Centre for Psychosocial Medicine, General Internal Medicine and Psychosomatics, University Hospital Heidelberg, Im Neuenheimer Feld 410, 69120 Heidelberg. Phone: 49.0.6221.56.38667; Email: Joe.simon@med.uni-heidelberg.de.

1. Berridge KC. Food reward: brain substrates of wanting and liking. Neurosci Biobehav Rev. 1996;20(1):1-25.

2. Berridge KC. The debate over dopamine's role in reward: the case for incentive salience. Psychopharmacology (Berl). 2007;191(3):391-431.

3. Knutson B, Fong GW, Adams CM, Varner JL, Hommer D. Dissociation of reward anticipation and outcome with event-related fMRI. Neuroreport. 2001;12(17):3683-3687.

4. Burger KS, Stice E. Elevated energy intake is correlated with hyperresponsivity in attentional, gustatory, and reward brain regions while anticipating palatable food receipt. Am J Clin Nutr. 2013;97(6):1188-1194.

5. Ferrario CR, et al. Homeostasis meets motivation in the battle to control food intake. J Neurosci. 2016;36(45):11469-11481

6. Murdaugh DL, Cox JE, Cook EW, Weller RE. fMRI reactivity to high-calorie food pictures predicts short- and long-term outcome in a weight-loss program. Neuroimage. 2012;59(3):2709-2721.

7. Simon JJ, et al. Impaired cross-talk between mesolimbic food reward processing and metabolic signaling predicts body mass index. Front Behav Neurosci. 2014;8:359.

8. Kringelbach ML. The human orbitofrontal cortex: linking reward to hedonic experience. Nat Rev Neurosci. 2005;6(9):691-702.

9. Rolls ET. Sensory processing in the brain related to the control of food intake. Proc Nutr Soc. 2007;66(1):96-112.

10. Rolls ET, Scott TR, Doty RL. Central taste anatomy and neurophysiology. In: Doty RL, ed. Handbook of olfaction and gustation New York: Marcel Dekker Inc.; 2003:679-705.

11. Simmons WK, Martin A, Barsalou LW. Pictures of appetizing foods activate gustatory cortices for taste and reward. Cereb Cortex. 2005;15(10):1602-1608.

12. Porubská K, Veit R, Preiss1 H, Fritsche A, Birbaumer N. Subjective feeling of appetite modulates brain activity: an fMRI study. Neuroimage. 2006;32(3):1273-1280.

13. Yokum S, Ng J, Stice E. Attentional bias to food images associated with elevated weight and future weight gain: an fMRI study. Obesity (Silver Spring). 2011;19(9):1775-1783

14. Stoeckel LE, Weller RE, Cook EW, Twieg DB, Knowlton RC, Cox JE. Widespread reward-system activation in obese women in response to pictures of high-calorie foods. Neuroimage. 2008;41(2):636-647.

15. Simon JJ, et al. Neural dissociation of food- and money-related reward processing using an abstract incentive delay task. Soc Cogn Affect Neurosci. 2015;10(8):1113-1120.

16. Uher R, et al. Effect of left prefrontal repetitive transcranial magnetic stimulation on food craving. Biol Psychiatry. 2005;58(10):840-842.

17. Gearhardt AN, Yokum S, Orr PT, Stice E, Corbin WR, Brownell KD. Neural correlates of food addiction. Arch Gen Psychiatry. 2011;68(8):808-816.

18. Kühn S, Gallinat J. The neural correlates of subjective pleasantness. Neuroimage. 2012;61(1):289-294.

19. Simon JJ, Skunde M, Walther S, Bendszus M, Herzog W, Friederich HC. Neural signature of food reward processing in bulimic-type eating disorders. Soc Cogn Affect Neurosci. 2016;11(9):1393-1401.

20. Siep N, Roefs A, Roebroeck A, Havermans R, Bonte ML, Jansen A. Hunger is the best spice: an fMRI study of the effects of 
attention, hunger and calorie content on food reward processing in the amygdala and orbitofrontal cortex. Behav Brain Res. 2009;198(1):149-158.

21. Morton GJ, Cummings DE, Baskin DG, Barsh GS, Schwartz MW. Central nervous system control of food intake and body weight. Nature. 2006;443(7109):289-295.

22. Fulton S, Woodside B, Shizgal P. Modulation of brain reward circuitry by leptin. Science. 2000;287(5450):125-128.

23. Berridge KC, Ho CY, Richard JM, DiFeliceantonio AG. The tempted brain eats: pleasure and desire circuits in obesity and eating disorders. Brain Res. 2010;1350:43-64.

24. Naleid AM, Grace MK, Cummings DE, Levine AS. Ghrelin induces feeding in the mesolimbic reward pathway between the ventral tegmental area and the nucleus accumbens. Peptides. 2005;26(11):2274-2279.

25. Kawahara Y, Kaneko F, Yamada M, Kishikawa Y, Kawahara H, Nishi A. Food reward-sensitive interaction of ghrelin and opioid receptor pathways in mesolimbic dopamine system. Neuropharmacology. 2013;67:395-402.

26. Skibicka KP, Shirazi RH, Hansson C, Dickson SL. Ghrelin interacts with neuropeptide Y Y1 and opioid receptors to increase food reward. Endocrinology. 2012;153(3):1194-1205.

27. Cone JJ, Roitman JD, Roitman MF. Ghrelin regulates phasic dopamine and nucleus accumbens signaling evoked by food-predictive stimuli. J Neurochem. 2015;133(6):844-856.

28. van der Plasse G, et al. Modulation of cue-induced firing of ventral tegmental area dopamine neurons by leptin and ghrelin. Int J Obes (Lond). 2015;39(12):1742-1749.

29. Cone JJ, McCutcheon JE, Roitman MF. Ghrelin acts as an interface between physiological state and phasic dopamine signaling J Neurosci. 2014;34(14):4905-4913

30. Woolley JD, Lee BS, Taha SA, Fields HL. Nucleus accumbens opioid signaling conditions short-term flavor preferences. Neuroscience. 2007;146(1):19-30.

31. Burghardt PR, Rothberg AE, Dykhuis KE, Burant CF, Zubieta JK. Endogenous opioid mechanisms are implicated in obesity and weight loss in humans. J Clin Endocrinol Metab. 2015;100(8):3193-3201.

32. Müller TD, et al. Ghrelin. Mol Metab. 2015;4(6):437-460.

33. Karra E, et al. A link between FTO, ghrelin, and impaired brain food-cue responsivity. J Clin Invest. 2013;123(8):3539-3551.

34. Perello M, Dickson SL. Ghrelin signalling on food reward: a salient link between the gut and the mesolimbic system. J Neuroendocrinol. 2015;27(6):424-434.

35. Malik S, McGlone F, Bedrossian D, Dagher A. Ghrelin modulates brain activity in areas that control appetitive behavior. Cell Metab. 2008;7(5):400-409.

36. Addolorato G, et al. Relationship between ghrelin levels, alcohol craving, and nutritional status in current alcoholic patients Alcohol Clin Exp Res. 2006;30(11):1933-1937.

37. Sun X, et al. The neural signature of satiation is associated with ghrelin response and triglyceride metabolism. Physiol Behav. 2014;136:63-73

38. Monteleone $\mathrm{P}$, et al. Hedonic eating is associated with increased peripheral levels of ghrelin and the endocannabinoid 2-arachidonoyl-glycerol in healthy humans: a pilot study. J Clin Endocrinol Metab. 2012;97(6):E917-E924.

39. Rigamonti AE, et al. Anticipatory and consummatory effects of (hedonic) chocolate intake are associated with increased circulating levels of the orexigenic peptide ghrelin and endocannabinoids in obese adults. Food Nutr Res. 2015;59:29678.

40. Monteleone P, Scognamiglio P, Monteleone AM, Perillo D, Canestrelli B, Maj M. Gastroenteric hormone responses to hedonic eating in healthy humans. Psychoneuroendocrinology. 2013;38(8):1435-1441.

41. Sescousse G, Caldú X, Segura B, Dreher JC. Processing of primary and secondary rewards: a quantitative meta-analysis and review of human functional neuroimaging studies. Neurosci Biobehav Rev. 2013;37(4):681-696.

42. Skibicka KP, Dickson SL. Ghrelin and food reward: the story of potential underlying substrates. Peptides. 2011;32(11):2265-2273.

43. García-García I, et al. Neural responses to visual food cues: insights from functional magnetic resonance imaging. Eur Eat Disord Rev. 2013;21(2):89-98.

44. Knutson B, Adams CM, Fong GW, Hommer D. Anticipation of increasing monetary reward selectively recruits nucleus accumbens. J Neurosci. 2001;21(16):RC159.

45. O'Doherty JP, Deichmann R, Critchley HD, Dolan RJ. Neural responses during anticipation of a primary taste reward. Neuron. 2002;33(5):815-826.

46. Zhang B, et al. Altered baseline brain activities before food intake in obese men: a resting state fMRI study. Neurosci Lett. 2015;584:156-161.

47. Hogenkamp PS, et al. Higher resting-state activity in reward-related brain circuits in obese versus normal-weight females independent of food intake. Int J Obes (Lond). 2016;40(11):1687-1692.

48. Lips MA, et al. Resting-state functional connectivity of brain regions involved in cognitive control, motivation, and reward is enhanced in obese females. Am J Clin Nutr. 2014;100(2):524-531.

49. Wierenga CE, et al. Hunger does not motivate reward in women remitted from anorexia nervosa. Biol Psychiatry. 2015;77(7):642-652.

50. Balodis IM, et al. Monetary reward processing in obese individuals with and without binge eating disorder. Biol Psychiatry. 2013;73(9):877-886

51. Dean Z, Horndasch S, Giannopoulos P, McCabe C. Enhanced neural response to anticipation, effort and consummation of reward and aversion during bupropion treatment. Psychol Med. 2016;46(11):2263-2274.

52. Stice E, Spoor S, Bohon C, Veldhuizen MG, Small DM. Relation of reward from food intake and anticipated food intake to obesity: a functional magnetic resonance imaging study. J Abnorm Psychol. 2008;117(4):924-935.

53. Stice E, Burger K, Yokum S. Caloric deprivation increases responsivity of attention and reward brain regions to intake, anticipated intake, and images of palatable foods. Neuroimage. 2013;67:322-330.

54. Aitken TJ, Greenfield VY, Wassum KM. Nucleus accumbens core dopamine signaling tracks the need-based motivational value of food-paired cues. J Neurochem. 2016;136(5):1026-1036.

55. Cabanac M. Sensory pleasure. Q Rev Biol. 1979;54(1):1-29.

56. Olszewski PK, Alsiö J, Schiöth HB, Levine AS. Opioids as facilitators of feeding: can any food be rewarding? Physiol Behav. 
2011;104(1):105-110.

57. Katsuura Y, Heckmann JA, Taha SA. mu-Opioid receptor stimulation in the nucleus accumbens elevates fatty tastant intake by increasing palatability and suppressing satiety signals. Am J Physiol Regul Integr Comp Physiol. 2011;301(1):R244-R254

58. Ziauddeen H, Farooqi IS, Fletcher PC. Obesity and the brain: how convincing is the addiction model? Nat Rev Neurosci. 2012;13(4):279-286.

59. Nummenmaa L, et al. Dorsal striatum and its limbic connectivity mediate abnormal anticipatory reward processing in obesity PLoS One. 2012;7(2):e31089.

60. Burger KS, Stice E. Variability in reward responsivity and obesity: evidence from brain imaging studies. Curr Drug Abuse Rev. 2011;4(3):182-189.

61. Overduin J, Figlewicz DP, Bennett-Jay J, Kittleson S, Cummings DE. Ghrelin increases the motivation to eat, but does not alter food palatability. Am J Physiol Regul Integr Comp Physiol. 2012;303(3):R259-R269.

62. King SJ, Isaacs AM, O'Farrell E, Abizaid A. Motivation to obtain preferred foods is enhanced by ghrelin in the ventral tegmental area. Horm Behav. 2011;60(5):572-580.

63. Davis JF, Choi DL, Clegg DJ, Benoit SC. Signaling through the ghrelin receptor modulates hippocampal function and meal anticipation in mice. Physiol Behav. 2011;103(1):39-43.

64. Walker AK, Ibia IE, Zigman JM. Disruption of cue-potentiated feeding in mice with blocked ghrelin signaling. Physiol Behav. 2012;108:34-43.

65. Hsu TM, Suarez AN, Kanoski SE. Ghrelin: A link between memory and ingestive behavior. Physiol Behav. 2016;162:10-17.

66. Eklund A, Nichols TE, Knutsson H. Cluster failure: Why fMRI inferences for spatial extent have inflated false-positive rates. Proc Natl Acad Sci USA. 2016;113(28):7900-7905.

67. Dreher JC, Schmidt PJ, Kohn P, Furman D, Rubinow D, Berman KF. Menstrual cycle phase modulates reward-related neural function in women. Proc Natl Acad Sci USA. 2007;104(7):2465-2470.

68. Berridge KC. 'Liking' and 'wanting' food rewards: brain substrates and roles in eating disorders. Physiol Behav. 2009;97(5):537-550.

69. Berridge KC, Kringelbach ML. Pleasure systems in the brain. Neuron. 2015;86(3):646-664.

70. Berridge KC, Robinson TE. Liking, wanting, and the incentive-sensitization theory of addiction. Am Psychol. 2016;71(8):670-679

71. Diekhof EK, Kaps L, Falkai P, Gruber O. The role of the human ventral striatum and the medial orbitofrontal cortex in the representation of reward magnitude - an activation likelihood estimation meta-analysis of neuroimaging studies of passive reward expectancy and outcome processing. Neuropsychologia. 2012;50(7):1252-1266.

72. Haber SN, Knutson B. The reward circuit: linking primate anatomy and human imaging. Neuropsychopharmacology. 2010;35(1):4-26.

73. Burke MV, Small DM. Effects of the modern food environment on striatal function, cognition and regulation of ingestive behavior. Curr Opin Behav Sci. 2016;9:97-105.

74. Wittchen H, Zaudig M, Fydrich T. [Structured Clinical Interview for DSM-IV Axis I and II. Handbook]. Göttingen: Hoegrefe; 1997.

75. Hautzinger M, Keller F, Kühner C. Beck Depressions-Inventar: BDI II. Revision. Frankfurt, Germany:Harcourt Test Services Frankfurt/Main; 2006.

76. Batterham RL, et al. Gut hormone PYY(3-36) physiologically inhibits food intake. Nature. 2002;418(6898):650-654.

77. Lehrl S, Triebig G, Fischer B. Multiple choice vocabulary test MWT as a valid and short test to estimate premorbid intelligence. Acta Neurol Scand. 1995;91(5):335-345.

78. Hilbert A, Tuschen-Caffier B, Ohms M. Eating disorder examination: Deutschsprachige Version des strukturierten Essstörungsinterviews. Diagnostica. 2004;50:98-106.

79. Hodgson RJ, Rachman S. Obsessional-compulsive complaints. Behav Res Ther. 1977;15(5):389-395.

80. Preuss UW, et al. [Psychometric evaluation of the German version of the Barratt Impulsiveness Scale]. Nervenarzt. 2008;79(3):305-319.

81. Cohen MS. Parametric analysis of fMRI data using linear systems methods. Neuroimage. 1997;6(2):93-103.

82. Brett M, Anton JL, Valabregue R, Poline JB. Region of interest analysis using the MarsBar toolbox for SPM 99. Neuroimage. 2002;16(2):S497.

83. Simon JJ, et al. Neural reward processing is modulated by approach- and avoidance-related personality traits. Neuroimage. 2010;49(2):1868-1874

84. Calhoun VD, Adali T, Pearlson GD, Pekar JJ. A method for making group inferences from functional MRI data using independent component analysis. Hum Brain Mapp. 2001;14(3):140-151.

85. Allen EA, et al. A baseline for the multivariate comparison of resting-state networks. Front Syst Neurosci. 2011;5:2.

86. Bell AJ, Sejnowski TJ. An information-maximization approach to blind separation and blind deconvolution. Neural Comput. 1995;7(6):1129-1159.

87. Erhardt EB, Rachakonda S, Bedrick EJ, Allen EA, Adali T, Calhoun VD. Comparison of multi-subject ICA methods for analysis of fMRI data. Hum Brain Mapp. 2011;32(12):2075-2095.

88. Maldjian JA, Laurienti PJ, Kraft RA, Burdette JH. An automated method for neuroanatomic and cytoarchitectonic atlas-based interrogation of fMRI data sets. Neuroimage. 2003;19(3):1233-1239.

89. Tzourio-Mazoyer N, et al. Automated anatomical labeling of activations in SPM using a macroscopic anatomical parcellation of the MNI MRI single-subject brain. Neuroimage. 2002;15(1):273-289. 